\title{
A framework for unified Dirac gauginos
}

\author{
Karim Benakli ${ }^{1, a}$ \\ ${ }^{1}$ Sorbonne Universités, UPMC Univ Paris 06, UMR 7589, LPTHE, F-75005, Paris, France \\ CNRS, UMR 7589, LPTHE, F-75005, Paris, France
}

\begin{abstract}
We identify the Minimal Dirac Gaugino Supersymmetric Standard Model (MDGSSM) as the minimal field content with Dirac gauginos allowing unification of gauge coupling. We stress that its parameter space describes also other most popular models as the MSSM, NMSSM and MRSSM. We discuss the generation of trilinear couplings in models of gauge mediation that has been overlooked in the past. We study the different source of Higgs mixings and constraints from the $\rho$ parameter. Finally, we provide new experimental limits on the masses of the scalar octets.
\end{abstract}

\section{Introduction}

The possibility of extending the MSSM to allow Dirac gaugino masses has been subject to many studies (see for example [1-23]. Multiple realisations have been proposed that differ by the fate of Rsymmetry, the presence or absence of additional states and interactions (for a short introduction see for example [14]). Here we argue that the field content of the MDGSSM is the minimal content of extra states to describe al these models that automatically preserve unification of gauge couplings while allowing the new couplings to the Higgs to enhance naturalness and allow the boundary conditions to be unified at a high energy scale.

The additional states compare to the MSSM are:

- A singlet, a triplet and an octet supermultiplets $\mathbf{S}, \mathbf{T}$ and $\mathbf{O}$, respectively, introduced to give the gauginos Dirac masses.

- There are extra vector-like charged states, subsequently called "fake leptons" [16] as they carry the same quantum numbers as the Standard Model leptons. They were introduced in order to restore the automatic gauge coupling unification that was spoiled by the addition of the adjoint representations of the Standard Model gauge group.

One of the important novelty of the Dirac gaugino models with respect to the NMSSM is its mixing with the Standard Model Higgs. This mixing is present at tree level due to the supersymmetric operator describing the Dirac gaugino bino mass leads to a modification of the $U(1)_{Y}$ D-term as

$$
D_{1}=D_{Y}^{(0)} \rightarrow D_{1}=-2 m_{1 D} S_{R}+D_{Y}^{(0)} \quad \text { with } \quad D_{Y}^{(0)}=-g^{\prime} \sum_{j} Y_{j} \varphi_{j}^{*} \varphi_{j}
$$

\footnotetext{
a e-mail: kbenakli@lpthe.jussieu.fr
} 
where $S_{R}$ is the real part of $S$ and $\varphi_{j}$ a scalar field with charge $Y_{j}$ under $U(1)_{Y}$. Upon elimination of the auxilliary fields, this implies an interaction of the form:

$$
g^{\prime} m_{1 D} S_{R}\left(\left|H_{u}^{0}\right|^{2}-\left|H_{d}^{0}\right|^{2}\right)
$$

thereof a tree-level induced mixing. However, as this can typically be compensated by the presence in the superpotential of a term of the form:

$$
W \supset \lambda_{S} S H_{u} H_{d}
$$

A precise evaluation of this mixing at the tree and one-loop level needs to be carried out carefully.

The parameter space is constrained by the requirements of stability of the vacuum avoiding existence of directions in the phase space of the model taking the fields expectation values to chargeand colour- breaking vacua. This is important due to trilinear terms, some have not been explicitly discussed in the existing literature while they are expected to be generically present in the model. This is the case for example of soft terms mixing three adjoint scalars that we will show that they are generated in models of gauge mediation. It is also constrained if one needs to keep couplings small enough if we want to preserve perturbativity up to the GUT scale. This restriction can be of course relaxed if one allows for Landau poles below the GUT scale. However, as one of the virtues of the MSSM was to predict perturbative unification of gauge couplings, and was one of the motivations for introducing the MDGSSM, we shall place emphasis on regions of the parameter space which respect this condition.

\section{The MDGSSM field content}

The MDGSSM field content can be seen as the minimal set providing the MSSM gauginos a Dirac mass while preserving two-loop unification and perturbativity of gauge couplings. We summarised it in Table 1. In addition to the chiral multiplets transforming under the adjoint representations of the gauge groups, it includes new fields charged under the lepton number global symmetry. They consist of extra Higgs-like doublets ${ }^{1} \mathbf{R}_{u}, \mathbf{R}_{d}$ as well as two pairs of vector-like right-handed electron superfields $\mathbf{E}_{1,2}^{\prime}$ in $(\mathbf{1}, \mathbf{1})_{1}$ and $\tilde{\mathbf{E}}_{1,2}^{\prime}$ in $(\mathbf{1}, \mathbf{1})_{-1}$. Such states are compatible with an $(S U(3))^{3}$ Grand Unification gauge group. This is the minimal set which enables a "natural" unification (unification without mass thresholds tuning) similar to the MSSM. The adjoint chiral multiplets contain new complex adjoint scalars, $S, T$ and $O$ :

$$
\begin{aligned}
& S=\frac{S_{R}+i S_{I}}{\sqrt{2}} \\
& T=\frac{1}{2 \sqrt{2}}\left(\begin{array}{cc}
T_{R}+i T_{I} & \sqrt{2}\left(T_{+R}+i T_{+I}\right) \\
\sqrt{2}\left(T_{-R}+i T_{-I}\right) & -\left(T_{R}+i T_{I}\right)
\end{array}\right) \\
& O^{(a)}=\frac{O_{R}^{(a)}+i O_{I}^{(a)}}{\sqrt{2}}
\end{aligned}
$$

where the $S_{R}, O_{R}^{(a)}, T_{R}, T_{-R}, T_{+R}$ and the $S_{I}, O_{I}^{(a)}, T_{I}, T_{-I}, T_{+I}$ are real scalars and pseudo-scalars, respectively.

\footnotetext{
${ }^{1}$ The hypercharges are opposite with respect to the Higgs doublet in the MSSM to match the MRSSM notation for the same fields.
} 


\begin{tabular}{|c|c|c|c|c|c|c|}
\hline Names & & Spin 0 & Spin $1 / 2$ & Spin 1 & $\left(S U(3), S U(2), U(1)_{Y}\right)$ & $R$-charge \\
\hline $\begin{array}{c}\text { Quarks } \\
\text { (×3 families) }\end{array}$ & $\begin{array}{c}\mathbf{Q} \\
\mathbf{U}^{\mathbf{c}} \\
\mathbf{D}^{\mathbf{c}}\end{array}$ & $\begin{array}{c}\tilde{Q}=\left(\tilde{u}_{L}, \tilde{d}_{L}\right) \\
\tilde{U}_{L}^{c} \\
\tilde{D}_{L}^{c}\end{array}$ & $\begin{array}{c}\left(u_{L}, d_{L}\right) \\
U_{L}^{c} \\
D_{L}^{c}\end{array}$ & & $\begin{array}{c}(\mathbf{3}, \mathbf{2}, 1 / 6) \\
(\overline{\mathbf{3}}, \mathbf{1},-2 / 3) \\
(\overline{\mathbf{3}}, \mathbf{1}, 1 / 3) \\
\end{array}$ & $\begin{array}{l}1 \\
0 \\
0\end{array}$ \\
\hline $\begin{array}{c}\text { Leptons } \\
(\times 3 \text { families })\end{array}$ & $\begin{array}{c}\mathbf{L} \\
\mathbf{E}^{\mathbf{c}}\end{array}$ & $\begin{array}{c}\left(\tilde{v}_{e L}, \tilde{e}_{L}\right) \\
\tilde{E}^{c}\end{array}$ & $\begin{array}{c}\left(v_{e L}, e_{L}\right) \\
E^{c}\end{array}$ & & $\begin{array}{c}(\mathbf{1}, \mathbf{2},-1 / 2) \\
(\mathbf{1}, \mathbf{1}, 1)\end{array}$ & $\begin{array}{l}1 \\
0\end{array}$ \\
\hline Higgs & $\begin{array}{l}\mathbf{H}_{\mathbf{u}} \\
\mathbf{H}_{\mathbf{d}}\end{array}$ & $\begin{array}{l}\left(H_{u}^{+}, H_{u}^{0}\right) \\
\left(H_{d}^{0}, H_{d}^{-}\right)\end{array}$ & $\begin{array}{l}\left(\tilde{H}_{u}^{+}, \tilde{H}_{u}^{0}\right) \\
\left(\tilde{H}_{d}^{0}, \tilde{H}_{d}^{-}\right)\end{array}$ & & $\begin{array}{l}(\mathbf{1}, \mathbf{2}, 1 / 2) \\
(\mathbf{1}, \mathbf{2},-1 / 2)\end{array}$ & $\begin{array}{l}1 \\
1\end{array}$ \\
\hline Gluons & $\mathbf{W}_{\mathbf{3} \alpha}$ & & $\begin{array}{c}\lambda_{3 \alpha} \\
{\left[\equiv \tilde{g}_{\alpha}\right]}\end{array}$ & $g$ & $(\mathbf{8}, \mathbf{1}, 0)$ & 1 \\
\hline $\mathrm{W}$ & $\mathbf{W}_{\mathbf{2} \alpha}$ & & $\begin{array}{c}\lambda_{2 \alpha} \\
{\left[\equiv \tilde{W}^{ \pm}, \tilde{W}^{0}\right]}\end{array}$ & $W^{ \pm}, W^{0}$ & $(\mathbf{1}, \mathbf{3}, 0)$ & 1 \\
\hline B & $\mathbf{W}_{1 \alpha}$ & & $\begin{array}{c}\lambda_{1 \alpha} \\
{[\equiv \tilde{B}]}\end{array}$ & $B$ & $(\mathbf{1}, \mathbf{1}, 0)$ & 1 \\
\hline DG-octet & O & $O$ & $\begin{array}{c}\chi_{g} \\
{\left[\equiv \tilde{g}^{\prime}\right]}\end{array}$ & & $(\mathbf{8}, \mathbf{1}, 0)$ & 0 \\
\hline DG-triplet & $\mathbf{T}$ & $\left\{T^{0}, T^{ \pm}\right\}$ & $\begin{aligned} & \left\{\chi_{T}^{0}, \chi_{T}^{ \pm}\right\} \\
{[\equiv} & \left.\left\{\tilde{W}^{\prime \pm}, \tilde{W}^{\prime 0}\right\}\right]\end{aligned}$ & & $(\mathbf{1}, 3,0)$ & 0 \\
\hline DG-singlet & $\mathbf{S}$ & $S$ & $\begin{array}{c}\chi_{S} \\
{\left[\equiv \tilde{B}^{\prime}\right]}\end{array}$ & & $(\mathbf{1}, \mathbf{1}, 0)$ & 0 \\
\hline $\begin{array}{l}\text { Higgs-like } \\
\text { leptons }\end{array}$ & $\begin{array}{l}\mathbf{R}_{\mathbf{u}} \\
\mathbf{R}_{\mathrm{d}}\end{array}$ & $\begin{array}{l}R_{u} \\
R_{d} \\
\end{array}$ & $\begin{array}{l}\tilde{R}_{u} \\
\tilde{R}_{d}\end{array}$ & & $\begin{array}{c}(\mathbf{1}, \mathbf{2},-1 / 2) \\
(\mathbf{1}, \mathbf{2}, 1 / 2)\end{array}$ & $\begin{array}{l}1 \\
1 \\
\end{array}$ \\
\hline $\begin{array}{c}\text { Fake } \\
\text { electrons }\end{array}$ & $\begin{array}{l}\hat{\mathbf{E}}(\times 2) \\
\hat{\mathbf{E}}^{\prime}(\times 2)\end{array}$ & $\begin{array}{l}\hat{E} \\
\hat{E}^{\prime}\end{array}$ & $\begin{array}{l}\hat{\hat{E}} \\
\hat{\tilde{E}}^{\prime}\end{array}$ & & $\begin{array}{l}(1,1,1) \\
(1,1,-1)\end{array}$ & $\begin{array}{l}0 \\
2\end{array}$ \\
\hline
\end{tabular}

Table 1: Chiral and gauge multiplet fields in the model.

The superpotential for these fields can be written as

$$
W=W_{\text {Yukawa }}+W_{D G}+W_{R V}
$$

where $W_{\text {Yukawa }}$ contains the usual MSSM Yukawas part

$$
W_{\text {Yukawa }}=Y_{u}^{i j} \mathbf{U}^{\mathbf{c}}{ }_{i} \mathbf{Q}_{j} \mathbf{H}_{u}-Y_{d}^{i j} \mathbf{D}^{\mathbf{c}}{ }_{i} \mathbf{Q}_{j} \mathbf{H}_{d}-Y_{e}^{i j} \mathbf{E}^{\mathbf{c}}{ }_{i} \mathbf{L}_{j} \mathbf{H}_{\mathbf{d}}
$$


$W_{D G}$ contains the $R$-symmetric (according to the choice of R-charges in table 1 ) contributions of the non-MSSM fields

$$
\begin{aligned}
W_{D G}= & \left(\mu+\lambda_{S} \mathbf{S}\right) \mathbf{H}_{\mathbf{d}} \mathbf{H}_{\mathbf{u}}+\sqrt{2} \lambda_{T} \mathbf{H}_{\mathbf{d}} \mathbf{T} \mathbf{H}_{\mathbf{u}} \\
& \left(\mu_{R}+\lambda_{S R} \mathbf{S}\right) \mathbf{R}_{\mathbf{u}} \mathbf{R}_{\mathbf{d}}+2 \lambda_{T R} \mathbf{R}_{\mathbf{u}} \mathbf{T} \mathbf{R}_{\mathbf{d}} \\
& +\left(\mu_{\hat{E} i j}+\lambda_{S E^{c} i j} \mathbf{S}\right) \hat{\mathbf{E}}_{i} \hat{\mathbf{E}}_{j}^{\prime}+\lambda_{S E i j} \mathbf{S E}_{i}^{\mathbf{c}} \hat{\mathbf{E}}_{j}^{\prime} \\
& +\lambda_{S L R i} \mathbf{S L}_{i} \mathbf{R}_{d}+2 \lambda_{T L R i} \mathbf{L}_{i} \mathbf{T} \mathbf{R}_{\mathbf{d}}-Y_{\hat{E} i} \mathbf{R}_{\mathbf{u}} \mathbf{H}_{\mathbf{d}} \hat{\mathbf{E}}_{i} \\
& -Y_{L F V}^{i j} \mathbf{L}_{\mathbf{i}} \cdot \mathbf{H}_{\mathbf{d}} \hat{\mathbf{E}}_{j}-Y_{E F V}^{j} \mathbf{R}_{\mathbf{u}} \mathbf{H}_{\mathbf{d}} \mathbf{E}^{\mathbf{c}}{ }_{j},
\end{aligned}
$$

while $W_{R V}$ gathers the R-symmetry violating terms

$$
\begin{aligned}
& W_{R V}= L \mathbf{S}+\frac{\hat{M}_{1}}{2} \mathbf{S}^{2}+\frac{\kappa}{3} \mathbf{S}^{3}+\hat{M}_{2} \operatorname{tr}(\mathbf{T T})+\hat{M}_{3} \operatorname{tr}(\mathbf{O O}) \\
&+\lambda_{S T} \mathbf{S t r}(\mathbf{T} \mathbf{T})+\lambda_{S O} \mathbf{S t r}(\mathbf{O O})+\frac{\kappa_{O}}{3} \operatorname{tr}(\mathbf{O O O})-Y_{\hat{E}^{\prime} i} \mathbf{R}_{\mathbf{d}} \mathbf{H}_{\mathbf{u}} \hat{\mathbf{E}}_{i}^{\prime} \\
& \underset{\mathrm{R}-\mathrm{symmetry}}{\longrightarrow} 0
\end{aligned}
$$

For the soft SUSY-breaking terms, from the MSSM we retain only the bilinear terms - i.e. conventional mass-squared terms and the $B_{\mu}$ term. All the scalar trilinear and Majorana gaugino mass terms violate R-symmetry; while for $B_{\mu}$ we suppose that, since R-symmetry is a chiral symmetry, we are breaking R-symmetry in the Higgs sector - and in fact it is only in combination with the superpotential terms $\mu, \lambda_{S}, \lambda_{T}$ that the R-symmetry is violated. Hence in principle we can have an entirely R-preserving supersymmetry-breaking sector.

The soft SUSY breaking terms beyond those of the MSSM consist of ${ }^{2}$ :

- Dirac gaugino masses:

$$
W_{\text {supersoft }}=\int d^{2} \theta \sqrt{2} \theta^{\alpha}\left[m_{D 1} \mathbf{S} W_{Y \alpha}+2 m_{D 2} \operatorname{tr}\left(\mathbf{T} W_{2 \alpha}\right)+2 m_{D 3} \operatorname{tr}\left(\mathbf{O} W_{3 \alpha}\right)\right] .
$$

- soft terms associated with the adjoint scalars

$$
\begin{aligned}
-\Delta \mathcal{L}_{\text {adjoints }}^{\text {scalar soft }}= & m_{S}^{2}|S|^{2}+\frac{1}{2} B_{S}\left(S^{2}+\text { h.c. }\right)+2 m_{T}^{2} \operatorname{tr}\left(T^{\dagger} T\right)+\left(B_{T} \operatorname{tr}(T T)+\text { h.c. }\right) \\
& +2 m_{O}^{2} \operatorname{tr}\left(O^{\dagger} O\right)+\left(B_{O} \operatorname{tr}(O O)+\text { h.c. }\right) \\
& +\left[T_{S} S H_{u} \cdot H_{d}+2 T_{T} H_{d} \cdot T H_{u}+\frac{1}{3} \kappa A_{K} S^{3}+t_{S} S+\text { h.c. }\right] \\
& +\left[T_{S O} S \operatorname{tr}\left(O^{2}\right)+T_{S T} S \operatorname{tr}\left(T^{2}\right)+\frac{1}{3} T_{O} \operatorname{tr}\left(O^{3}\right)+\text { h.c. }\right]
\end{aligned}
$$

The terms on the last line have generally been neglected, but will play an important role in this work.

- soft terms involving the new vector-like leptons:

$$
\begin{aligned}
-\Delta \mathcal{L}_{\text {vector-like }}^{\text {scalar soft }}= & m_{R_{u}}^{2}\left|R_{u}\right|^{2}+m_{R_{d}}^{2}\left|R_{d}\right|^{2}+\left[B_{R} R_{d} R_{u}+\text { h.c. }\right] \\
& +\hat{E}_{i}\left(m_{\hat{E}}^{2}\right)_{j}^{i} \hat{E}^{j}+\hat{E}^{\prime i}\left(m_{\hat{E}^{\prime}}^{2}\right)_{i}^{j} \hat{E}_{j}^{\prime}+\left[B_{\hat{E}}^{i j} \hat{E}_{i} \hat{E}_{j}^{\prime}+\text { h.c. }\right] \\
& +\left[T_{S E}^{i j} S \hat{E}_{i} \hat{E}_{j}^{\prime}+T_{S R} S R_{d} R_{u}+\text { h.c. }\right]
\end{aligned}
$$

\footnotetext{
${ }^{2}$ We suppress gauge indices while retaining generation indices and denote the complex conjugation of fields by upper versus lower indices.
} 
Let us highlight that in an R-symmetry conserving model, one cannot simultaneously have the trilinears $T_{S E}$ (respectively $T_{S R}$ ) from (2.8) and the superpotential couplings $\lambda_{S E}$ (respectively $\lambda_{S R}$ ) from (2.4) as each term requires a different R-charge for the fields $\hat{E}$ and $\hat{E}^{\prime}$ (respectively $R_{u}$ and $R_{d}$ ) to be R-invariant.

We consider now the scalar mass matrix. We use the notation

$$
\begin{aligned}
& \tilde{m}_{S}^{2}=\tilde{m}_{S R}^{2}+\lambda_{S}^{2} \frac{v^{2}}{2} \\
& \tilde{m}_{T}^{2}=\tilde{m}_{T R}^{2}+\lambda_{T}^{2} \frac{v^{2}}{2},
\end{aligned}
$$

where the effective masses for the real parts of $S$ and $T$ read:

$$
\tilde{m}_{S R}^{2}=m_{S}^{2}+4 m_{1 D}^{2}+B_{S}, \quad \tilde{m}_{T R}^{2}=m_{T}^{2}+4 m_{2 D}^{2}+B_{T} .
$$

Then, at tree level the scalar mass matrix in the basis $\left\{h, H, S_{R}, T_{R}^{0}\right\}$ is [15]:

$$
\left(\begin{array}{cccc}
M_{Z}^{2}+\Delta_{h} s_{2 \beta}^{2} & \Delta_{h} s_{2 \beta} c_{2 \beta} & \Delta_{h S} & \Delta_{h T} \\
\Delta_{h} s_{2 \beta} c_{2 \beta} & M_{A}^{2}-\Delta_{h} s_{2 \beta}^{2} & \Delta_{H S} & \Delta_{H T} \\
\Delta_{h S} & \Delta_{H S} & \tilde{m}_{S}^{2} & \lambda_{S} \lambda_{T} \frac{v^{2}}{2} \\
\Delta_{h T} & \Delta_{H T} & \lambda_{S} \lambda_{T} \frac{v^{2}}{2} & \tilde{m}_{T}^{2}
\end{array}\right)
$$

where we have defined:

$$
\Delta_{h}=\frac{v^{2}}{2}\left(\lambda_{S}^{2}+\lambda_{T}^{2}\right)-M_{Z}^{2}
$$

which vanishes when $\lambda_{S}$ and $\lambda_{T}$ take their $N=2$ values,

$$
\Delta_{h S}=-2 \frac{v_{S}}{v} \tilde{m}_{S R}^{2}, \quad \Delta_{h T}=-2 \frac{v_{T}}{v} \tilde{m}_{T R}^{2}
$$

and

$$
\Delta_{H S}=g^{\prime} m_{1 D} v s_{2 \beta}, \quad \Delta_{H T}=-g m_{2 D} v s_{2 \beta} .
$$

This matrix is diagonalised by the mixing matrix $S_{i j}$. Of particular interest will be $S_{11}$ which measures if the lightest scalar eigenstate is Standard Model Higgs like, and $S_{13}$ which measures the proportion of the scalar singlet $S_{R}$ in this lightest eigenstate.

\section{Higgs mixing}

The singlet $S$ is that it will in general mix with the Higgs eigenstates. This will lead to tree-level decays of $S$ into tops, $W, Z$ or Higgs which are experimentally constrained. The minimisation condition of $v_{S}$ on the off-diagonal element $\Delta_{h S}$ of the scalar mass matrix given in (2.13) leads to (see [13])

$$
\begin{aligned}
\Delta_{h S} & =v\left[v_{S} \lambda_{S}^{2}-g^{\prime} m_{1 D} c_{2 \beta}+\sqrt{2} \lambda_{S} \mu+\lambda_{S} \lambda_{T} v_{T}\right] \\
& =v\left[\sqrt{2} \lambda_{S} \tilde{\mu}-g^{\prime} m_{1 D} c_{2 \beta}\right],
\end{aligned}
$$

where we used the effective mass parameter

$$
\tilde{\mu}=\mu+\frac{1}{\sqrt{2}}\left(\lambda_{S} v_{S}+\lambda_{T} v_{T}\right) .
$$


From this basic analytical calculation, we see that we can minimise the tree-level mixing by choosing:

$$
\lambda_{S} \sim \frac{g_{Y} m_{1 D} c_{2 \beta}}{\sqrt{2} \tilde{\mu}} .
$$

In general, this relation will be modified at one-loop, but the property that one value of $\lambda_{S}$ is favoured will remain.

Also, the v.e.v. of $T$ gives a contribution to the $W$ boson mass and the electroweak precision data give bounds on it. The induced correction to the $\rho$-parameter:

$$
\rho \equiv \frac{M_{W}^{2}}{c_{\theta_{W}}^{2} M_{Z}^{2}}=1+\Delta \rho,
$$

with $\Delta \rho$ is given analytically at tree-level by ([13])

$$
\Delta \rho \sim \frac{4 v_{T}^{2}}{v^{2}}
$$

where $v$ is the usual Standard Model Higgs v.e.v.. In order to be below the experimental constraints, we need $\Delta \rho \lesssim(4.2 \pm 2.7) \times 10^{-4},([13]-$ see also [24, 25] - ). At tree level, we have

$$
v_{T} \simeq \frac{v^{2}}{2 \tilde{m}_{T R}^{2}}\left[-g m_{2 D} c_{2 \beta}-\sqrt{2} \tilde{\mu} \lambda_{T}\right],
$$

with $\tilde{m}_{T R}^{2}=m_{T}^{2}+4 m_{2 D}^{2}+B_{T}$, therefore, small $\Delta \rho$ require large triplet Dirac and soft masses. This requirement can often be at odd with naturalness which prefers smaller triplet masses. Indeed, radiative corrections induced by the adjoint triplet scalars to $m_{H_{u, d}}^{2}$ are [13]:

$$
\delta m_{H_{u, d}}^{2} \supset-\frac{1}{16 \pi^{2}}\left(2 \lambda_{T}^{2} m_{T}^{2}\right) \log \left\{\frac{\Lambda}{\mathrm{TeV}}\right\},
$$

with $\Lambda$ the UV cut-off, $m_{H_{u, d}}^{2}, m_{T}^{2}$ the squared masses for Higgses and scalar triplet $T$, and $\lambda_{T}$ the coupling defined in (2.4). For $\Lambda$ at the Planck scale, requiring a fine-tuning $\Delta_{T}=\delta m_{H}^{2} / m_{H}^{2}$ better than $10 \%$ finally gives us

$$
m_{T} \lesssim \frac{1}{\lambda_{T}} 450 \mathrm{GeV}
$$

\section{Trilinear couplings}

Previous studies of Dirac gaugino models have generally neglected the phenomenology of adjoint self-coupling terms, with an exception being a superpotential term $\frac{\kappa}{3} S^{3}$ used in [13] to generate $\mu / B_{\mu}$ as in the NMSSM, and a recent brief discussion in [26]. In the case of superpotential terms such as $\lambda_{S O}$ these can be neglected when considering an R-symmetric visible sector; however, trilinear soft couplings such as $T_{S O}, T_{O}$ (see (2.7)) are always allowed. It is therefore interesting to consider what values we expect from models of supersymmetry-breaking mediation.

Starting with a spurion analysis where supersymmetry is broken by either a D-term $D$ or F-term $F$, then if the mediating dynamics is at a scale $M$ the terms in our effective Lagrangian should be given by powers of $\frac{D}{M}, \frac{F}{M}, \frac{D}{M^{2}}, \frac{F}{M^{2}}$ with appropriate factors of couplings and $\kappa_{l} \equiv 1 / 16 \pi^{2}$. Furthermore, quartic and higher-order couplings - which are "hard" SUSY-breaking parameters - are always generated, but 
do not lead to quadratic divergences because they appear suppressed by powers of the scale $M$ which is the cutoff of our effective theory. Important in this work are the quartics such as $\mathcal{L} \supset \frac{\lambda_{4 S}}{24} S^{4}$ which must have size $\lambda_{4 S} \sim \kappa_{l}^{p}\left(\frac{D}{M^{2}}\right)^{q}$ for some integer $p, q$ (or similarly for F-terms with even $q$ )); taking $p=1, q=1$ for a D-term we naively have a quadratic divergence in the scalar mass proportional to $\lambda_{4 S}$ but this yields $\Delta m_{S}^{2} \sim \kappa_{l} \lambda_{4 S} M^{2} \sim \kappa_{l}^{2} D \ll M^{2}$, while for $q=2$ we have $\kappa_{l}^{2} \frac{D^{2}}{M^{2}}$. In fact, this tells us that the case $q=1$ is special because it implies a much larger correction at one loop than the direct mass, and could therefore destabilise the calculation. We shall return to this below.

As a first observation, if the mediation is by gravity, then $M$ should be identified with the Planck scale (unless there is significant sequestering) and we should only consider the leading order terms. We would therefore require the quantum gravity theory to give us the terms $T_{S O}, T_{O}$ at leading order $D / M, F / M$ and the quartics must, by the above reasoning, be negligible.

On the other hand, in the case of low-scale supersymmetry breaking - where it was argued in [21] that this requires Dirac gauginos $-\sqrt{F} \sim \sqrt{D} \sim M \sim \mathrm{TeV}$, and we generate all terms at a similar order, which would include $T_{S O}, T_{O}$. However, the phenomenology is significantly changed by the presence of higher-dimensional operators and the goldstino couplings [27] and, since it is difficult to reconcile with perturbative unification, we shall not discuss this further here.

Finally, for gauge mediation $M$ could be as small as $\sqrt{F}$ or $\sqrt{D}$ but there is no a priori upper limit on $M$ until we choose a particular quantum gravity embedding. The Dirac gaugino masses are expected to be generated at one loop and be of order $\kappa_{l} \frac{D}{M}$ or $\kappa_{l} \frac{F^{2}}{M^{3}}$. For F-term breaking the standard gauge-mediation soft mass-squareds for the squarks/sleptons are of order $\kappa_{l}^{2} \frac{F^{2}}{M^{2}}$, while in Dterm breaking they may be suppressed. Therefore if we imagine that $\kappa_{l} \frac{D}{M} \sim \mathrm{TeV}$, then for terms $\kappa_{l} \frac{D^{2}}{M^{3}}$ to be significant we would need $D \sim M^{2}$ and furthermore $M \sim 100 \mathrm{TeV}$.

\subsection{Adjoint couplings in gauge mediation}

One of the most interesting issues in the construction of gauge mediation models with Dirac gaugino masses has been that of the adjoint scalar masses: in the simplest realisation, only a B-type masssquared $\mathcal{L} \supset-\frac{1}{2} B_{\Sigma} \Sigma^{2}$ is generated at leading order in $D / M^{2}$, and not a conventional mass-squared $\mathcal{L} \supset-m_{\Sigma}^{2}|\Sigma|^{2}$. This happens for one pair of vector-like messengers $Q, \tilde{Q}$ having charges under a hidden $U(1)$ of $+1,-1$, where the $U(1)$ obtains a D-term. This was noticed from the earliest models [3,5] with the original proposed solution being to add a supersymmetric mass for the adjoint - which would also violate the R-symmetry and generate Majorana masses for the gauginos, with a see-saw effect. However, an alternative solution was found to be to introduce additional messenger states with nondiagonal couplings to either the adjoints (in the D-term case) [10, 12] or an F-term spurion [8, 10, 12]; in the D-term case this requires the couplings to violate the $U(1)$-charges. In [12] examples were given where the ratio of B-type to conventional masses is arbitrary. The general ansatz was to couple the adjoint to messenger fields $Q_{i}, \tilde{Q}_{j}$ and to possible F-term spurions $X$ via superpotential couplings

$$
W \supset M Q_{i} \tilde{Q}_{i}+\lambda_{i j} Q_{i} \Sigma \tilde{Q}_{j}+\mu_{i j} X Q_{i} \tilde{Q}_{j}
$$

and D-terms via charges $e_{i}, \tilde{e}_{i}$ which we can write as a matrix $e_{i \bar{j}}\left(Q_{i} Q_{j}^{*}-\tilde{Q}_{i} \tilde{Q}_{j}^{*}\right)$.

More in the spirit of the earlier works, the issue was rephrased in the language of effective operators in $[26,28,29]$, where it was claimed that the explanation for the absence of conventional mass-squared terms for the adjoints at leading order is that the operator responsible for the generation of a leading-order mass-squared term should be

$$
\mathcal{L} \supset \int d^{4} \theta \frac{1}{M^{2}}\left[\psi^{\dagger} e^{q V} \psi+\tilde{\psi}^{\dagger} e^{-q V} \tilde{\psi}\right] \Sigma^{\dagger} \Sigma,
$$


where $\psi, \tilde{\psi}$ are a pair of fields charged under the hidden $U(1)$ with charges $\pm q$ which obtain v.e.v.s (and thus generate a contribution to the hidden D-term). The above operator is generated by including terms in the superpotential that mix the messengers $Q, \tilde{Q}$ with other pairs of fields $N, \tilde{N}$ which are neutral (or at least have different charges) under the hidden $U(1)$, so that the v.e.v.s of $\psi, \tilde{\psi}$ generate messenger mixing terms. This is clearly nearly equivalent to the above ansatz, and can be written in the form

$$
W \supset M_{i j} Q_{i} \tilde{Q}_{j}+\lambda_{i} Q_{i} \Sigma \tilde{Q}_{i}
$$

where we now write the mass terms as violating the $U(1)$ charges instead.

If we start with the case of no couplings/mass mixing terms that violate the $U(1)$ D-term charges, we shall first give a simple proof that the conventional mass term $|\Sigma|^{2}$ vanishes at leading order for any number of messengers, and then look at higher-order terms. Considering first the visible gauge group to be $U(1)$, we have the effective potential contribution from the messenger scalars (since the fermion potential is independent of $D$ ):

$$
\begin{aligned}
V & =\int \frac{d^{d} q}{(2 \pi)^{d}} \operatorname{tr} \log \left(q^{2}+\mathcal{M}_{Q}^{2}+D e\right)+\operatorname{tr} \log \left(q^{2}+\mathcal{M}_{\tilde{Q}}^{2}-D e\right) \\
& \equiv V_{+}+V_{-}
\end{aligned}
$$

Here we have $\mathcal{M}_{Q}^{2}=(M+\lambda \Sigma)\left(M^{\dagger}+\lambda^{\dagger} \bar{\Sigma}\right), \mathcal{M}_{\tilde{Q}}^{2}=\left(M^{\dagger}+\lambda^{\dagger} \bar{\Sigma}\right)(M+\lambda \Sigma)\left(M^{\dagger}+\lambda^{\dagger} \bar{\Sigma}\right)$ are the supersymmetric mass-squared matrices. Now, if we take the couplings to preserve the $U(1)$ charges then we can write the

$$
\begin{aligned}
V_{+} & =D e \int \frac{d^{d} q}{(2 \pi)^{d}} \operatorname{tr}\left(\frac{1}{q^{2}+\mathcal{M}_{Q}^{2}}\right)-\frac{1}{2} D^{2} e^{2} \int \frac{d^{d} q}{(2 \pi)^{d}} \operatorname{tr}\left(\frac{1}{q^{2}+\mathcal{M}_{Q}^{2}}\right)^{2}+O\left(D^{3}\right) \\
\rightarrow 16 \pi^{2} V & =D^{2} e^{2} \operatorname{tr}\left(\log \mathcal{M}_{Q}^{2} / \mu^{2}\right)+O\left(D^{4}\right)
\end{aligned}
$$

since the eigenvalues of $\mathcal{M}_{Q}^{2}$ and $\mathcal{M}_{\tilde{Q}}^{2}$ are equal. Next, by taking the derivative with respect to $\Sigma$ we find only a holomorphic function of $\Sigma$ :

$$
\begin{aligned}
16 \pi^{2} \frac{\partial V}{\partial \Sigma} & =D^{2} e^{2} \operatorname{tr}\left([M+\lambda \Sigma]^{-1} \lambda\right)+O\left(D^{4}\right) \\
\rightarrow V & =\frac{D^{2} e^{2}}{16 \pi^{2}}\left[\operatorname{tr}\left(\log M M^{\dagger} / \mu^{2}\right)+\tilde{V}(\Sigma)+\overline{\tilde{V}}(\bar{\Sigma})\right]+O\left(D^{4}\right) .
\end{aligned}
$$

As an example, consider the simple model of a single messenger where the matrices become numbers; then we have

$$
\tilde{V}(\Sigma)=-\sum_{n=1}^{\infty} \frac{1}{n}\left(\frac{-\lambda \Sigma}{M}\right)^{n}
$$

This potential manifestly has trilinear and quartic couplings, although at order $\frac{D^{2}}{M^{3}}, \frac{D^{2}}{M^{4}}$ respectively. Indeed, if we continue with the ansatz (4.1) then it is easy to see that there are no terms of linear order in $D$, because $\mathcal{M}_{Q}^{2}=(M+\lambda \Sigma)\left(M+\lambda^{\dagger} \Sigma^{\dagger}\right)=\mathcal{M}_{\tilde{Q}}^{2}$ and

$$
V=D \int \frac{d^{d} q}{(2 \pi)^{d}} \operatorname{tr}\left\{\left(\left[q^{2}+\mathcal{M}_{Q}^{2}\right]^{-1}-\left[q^{2}+\mathcal{M}_{\tilde{Q}}^{2}\right]^{-1}\right) e\right\}+O\left(D^{2}\right) .
$$


Hence to have large cubic interactions we should start from ansatz (4.3). In this way, in order to have an interesting phenomenology we require either $D \sim M^{2}$ with both at a low scale, or we require (as proposed in [28]) that

$$
B_{\Sigma}<m_{\Sigma}^{2} \sim a D+b \frac{D^{2}}{M^{2}}
$$

with some cancellation between the two terms so that we can have $m_{\Sigma} \sim T_{S O}$. Note that once we take this ansatz with non-zero mixing between the messengers and $[M, e] \neq 0$ we typically generate trilinear terms in the potential - but also tadpoles. The issue of tadpoles is then easily circumvented by embedding the coupling of the singlet adjoint $S$ to the $S U(3)$ and $S U(2)$ adjoints into the generator $T^{Y}=\frac{1}{\sqrt{60}} \operatorname{diag}(2,2,2,-3,-3)$. This then also means that the couplings of the singlet adjoint $S$ are related to those of $T$ and $O$; for example, for $T_{S O}$, if we have calculated the coupling for $U(1)$ messengers as being $\mathcal{L} \supset \frac{1}{6} T_{\Sigma} \Sigma^{3}$, then we have

$$
\begin{aligned}
T_{S O} \delta^{a b} & =T_{\Sigma} \operatorname{tr}\left(T^{Y} T_{3}^{a} T_{3}^{b}\right) \\
& =\frac{1}{\sqrt{15}} T_{\Sigma} \delta^{a b}
\end{aligned}
$$

where $T_{3}^{a}, T_{3}^{b}$ are $S U(3)$ generators. However, exploring sets of messengers which give these desired properties with sufficiently large trilinear couplings and exploring the vacuum stability of the total system would be very interesting, but is beyond the scope of this work.

\section{Octets production}

Consider the case when either the scalar or pseudoscalar colour octets are lighter than a TeV. Even though such light scalars should be copiously produced in pairs at both 8 and $13 \mathrm{TeV}$, as shown in figure 1, their decays are loop suppressed and this inhibits single production.

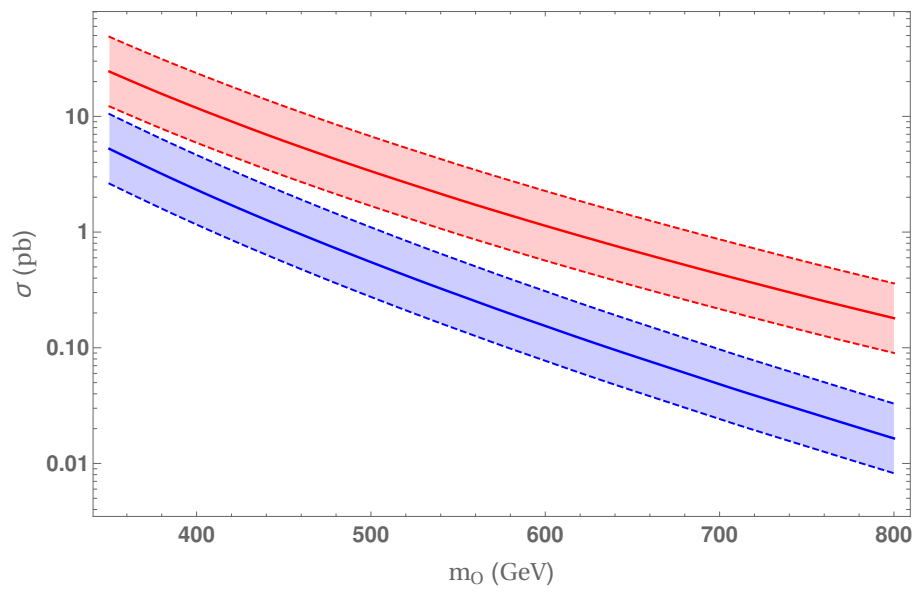

Figure 1: Pair production cross-section of octets at tree-level, at $8 \mathrm{TeV}$ (blue, lower curves) and $13 \mathrm{TeV}$ (red, upper curves). The bands indicate a variation of a factor of 2 each way relative to the values obtained in MadGraph. 
Since current limits place all squarks above about $800 \mathrm{GeV}$, then, as discussed in [30], the octets decay only to gluons and quarks - in particular almost entirely top quarks. This means that the possible signatures are four jets, dijet/ditop searches, and four tops. Up until relatively recently the constraints on them were rather weak, with dijets providing no constraint, and a mild constraint from ditops [31]. However, now the four top channel is particularly important: [32] placed a limit of $32 \mathrm{fb}$ at $8 \mathrm{TeV}$, and [33] found $370 \mathrm{fb}$ for Standard-Model-like kinematics, or $140 \mathrm{fb}$ with and EFT pointlike interaction, at $13 \mathrm{TeV}$.

We consider the cross-section times branching ratio approach, taking the most conservative values of twice the tree-level cross-section (i.e. a K-factor of 2) and a limit at $13 \mathrm{TeV}$ of $140 \mathrm{fb}$. To compute the branching ratio into four tops, we require the widths into gluons and tops. Expressions given in [30] used complex octets, which is not appropriate for our case where the necessarily large gluino mass causes a large splitting. Instead we use the expressions presented in [27].

The first important observation is that the pseudoscalar octet does not couple to gluons, and so pair production of pseudoscalar octets yields only four-top events, and by our above criteria excludes pseudoscalars below about $880 \mathrm{GeV}$ by the $13 \mathrm{TeV}$ data. These are therefore less interesting for our analysis.

On the other hand, the scalar octet couples to squarks via its D-term coupling, and so couples to gluons. Since it couples to all coloured squarks, this can potentially be large. However, to be very conservative, we show production times branching ratio of four-tops via scalar octets in figure 2 at 8 and $13 \mathrm{TeV}$ with the limits shown using a K-factor of 2, as we vary the octet mass and for three different values of the Dirac gluino mass, where the first two generations of squarks are decoupled (i.e. heavy and degenerate). To produce these, we take left-handed stops and sbottoms of $1200 \mathrm{GeV}$, righthanded stops of $800 \mathrm{GeV}$, and decoupled right-handed sbottoms (at $4 \mathrm{TeV}$ ). We neglect all squark mixing (which is a good approximation in this model). Since the couplings involve a cancellation between left- and right-handed squarks, this is very conservative: if we took heavier left-handed squarks, we would enhance the gluon rate relative to the top rate (because it has a contribution from sbottoms as well as stops) weakening the bounds.

We conclude that for $2.5 \mathrm{GeV}$ gluinos, the octet scalars must be heavier than $500 \mathrm{GeV}$; but for 3 $\mathrm{TeV}$ gluinos there is no constraint.
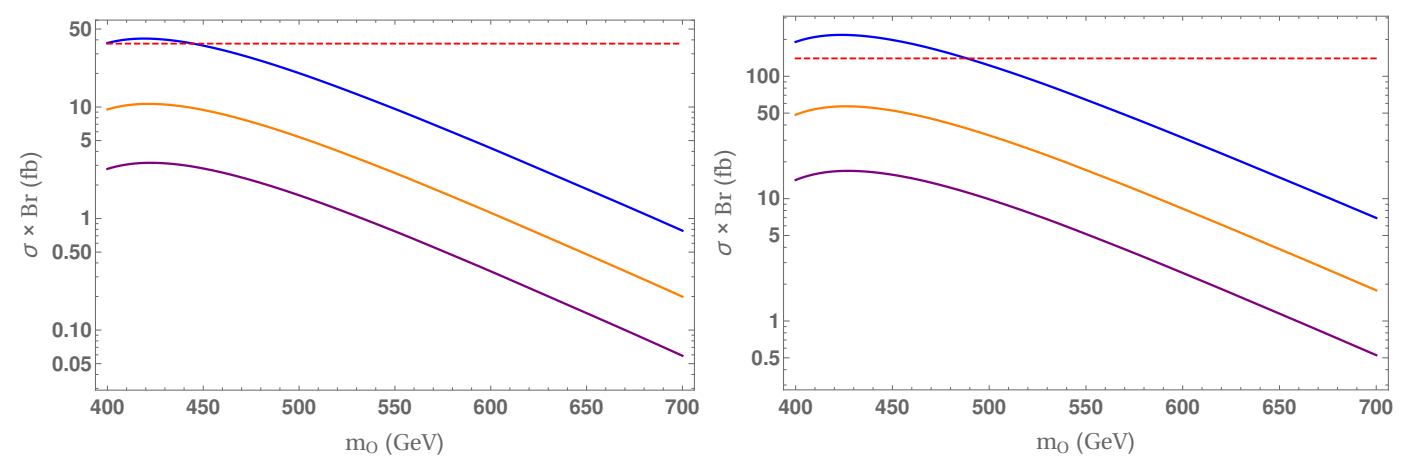

Figure 2: Four-top production times branching ratio from scalar colour octets as a function of the octet mass, for gluino masses of $2.5 \mathrm{TeV}$ (upper curve, blue), $3 \mathrm{TeV}$ (middle curve, orange) and $3.5 \mathrm{TeV}$ (lower curve, purple). The experimental limit is shown as the dashed red horizontal line. The left plot is computed for $\sqrt{s}=8 \mathrm{TeV}$, and right is for $\sqrt{s}=13 \mathrm{TeV}$. 


\section{Acknowledgments}

This work is supported by the European Research Council (ERC) under the Advanced Grant Higgs@LHC (ERC-2012-ADG20120216-321133), the support of the Agence Nationale de Recherche under grant ANR-15-CE31-0002 "HiggsAutomator", the Labex "Institut Lagrange de Paris" (ANR-11-IDEX-0004-02, ANR-10-LABX-63).

\section{References}

[1] P. Fayet, Phys.Lett. B78 417 (1978)

[2] L. J. Hall and L. Randall, Nucl. Phys. B352 289 (1991)

[3] P. J. Fox, A. E. Nelson, and N. Weiner, JHEP 0208035 (2002)

[4] A. E. Nelson, N. Rius, V. Sanz, and M. Unsal, JHEP 08039 (2002)

[5] I. Antoniadis, K. Benakli, A. Delgado, and M. Quiros, Adv. Stud. Theor. Phys. 2645 (2008)

[6] I. Antoniadis, K. Benakli, A. Delgado, M. Quiros and M. Tuckmantel, Nucl. Phys. B 744156 (2006)

[7] G. D. Kribs, E. Poppitz, and N. Weiner, Phys. Rev. D78 055010 (2008)

[8] S. D. L. Amigo, A. E. Blechman, P. J. Fox, and E. Poppitz, JHEP 01018 (2009)

[9] G. Belanger, K. Benakli, M. Goodsell, C. Moura, and A. Pukhov, JCAP 0908027 (2009)

[10] K. Benakli and M. D. Goodsell, Nucl. Phys. B816 185 (2009)

[11] K. Benakli and M. D. Goodsell, Nucl. Phys. B830 315 (2010)

[12] K. Benakli and M. D. Goodsell, Nucl. Phys. B840 1 (2010)

[13] K. Benakli, M. D. Goodsell, and A.-K. Maier, Nucl. Phys. B851 445 (2011)

[14] K. Benakli, Fortsch.Phys. 591079 (2011)

[15] K. Benakli, M. D. Goodsell, and F. Staub, JHEP 06073 (2013)

[16] K. Benakli, L. Darmé, M. D. Goodsell, and P. Slavich, JHEP 05113 (2014)

[17] K. Benakli, M. Goodsell, F. Staub, and W. Porod, Phys. Rev. D90 no. 4, 045017 (2014)

[18] E. Dudas, M. Goodsell, L. Heurtier, and P. Tziveloglou, Nucl. Phys. $B 884632$ (2014)

[19] K. Benakli, L. Darmé and M. D. Goodsell, JHEP 1511100 (2015)

[20] S. Abel and M. Goodsell, JHEP 06064 (2011)

[21] T. Gherghetta and A. Pomarol, JHEP 12069 (2011)

[22] S. P. Martin, Phys. Rev. D92 no. 3, 035004 (2015)

[23] R. Davies, J. March-Russell, and M. McCullough, JHEP 1104108 (2011)

[24] E. Bertuzzo, C. Frugiuele, T. Gregoire, and E. Ponton, JHEP 04089 (2015)

[25] H. Beauchesne and T. Gregoire, JHEP 05051 (2014)

[26] L. M. Carpenter and J. Goodman, JHEP 07107 (2015)

[27] M. D. Goodsell and P. Tziveloglou, Nucl. Phys. B889 650 (2014)

[28] C. Csaki, J. Goodman, R. Pavesi, and Y. Shirman, Phys. Rev. D89 no. 5, 055005 (2014)

[29] L. M. Carpenter, JHEP 09102 (2012)

[30] S. Y. Choi, M. Drees, J. Kalinowski, J. M. Kim, E. Popenda, and P. M. Zerwas, Phys. Lett. B672 246 (2009)

[31] CMS Collaboration, S. Chatrchyan et al., Phys. Rev. Lett. 111 no. 21, 211804 (2013), [Erratum: Phys. Rev. Lett.112, no.11, 119903 (2014)].

[32] CMS Collaboration, V. Khachatryan et al., JHEP 11154 (2014)

[33] T. A. collaboration, "Search for production of vector-like top quark pairs and of four top quarks in the lepton-plus-jets final state in $p p$ collisions at $\sqrt{s}=13 \mathrm{TeV}$ with the ATLAS detector,". 